\title{
How complex do models need to be to predict dispersal of threatened species through matrix habitats?
}

\author{
Brian R. Hudgens, ${ }^{1,6}$ William F. Morris, ${ }^{2}$ Nick M. Haddad,${ }^{3}$ William R. Fields,,${ }^{3}$ John W. Wilson, ${ }^{3}$ \\ Daniel Kuefler, ${ }^{4}$ And Todd Jobe ${ }^{5}$ \\ ${ }^{1}$ Institute for Wildlife Studies, 55 Ericson Court, Suite 1, Arcata, California 95518 USA \\ ${ }^{2}$ Department of Biology, Duke University, Box 90338, Durham, North Carolina 27708-0338 USA \\ ${ }^{3}$ Department of Biology, Box 7617, North Carolina State University, Raleigh, North Carolina 27695-7617 USA \\ ${ }^{4}$ Department of Integrative Biology, University of Guelph, Science Complex, 50 Stone Road E., Guelph, Ontario N1G 2 W1 Canada \\ ${ }^{5}$ Department of Geography, University of North Carolina, Chapel Hill, North Carolina 27599 USA
}

\begin{abstract}
Persistence of species in fragmented landscapes depends on dispersal among suitable breeding sites, and dispersal is often influenced by the "matrix" habitats that lie between breeding sites. However, measuring effects of different matrix habitats on movement and incorporating those differences into spatially explicit models to predict dispersal is costly in terms of time and financial resources. Hence a key question for conservation managers is: Do more costly, complex movement models yield more accurate dispersal predictions? We compared the abilities of a range of movement models, from simple to complex, to predict the dispersal of an endangered butterfly, the Saint Francis' satyr (Neonympha mitchellii francisci). The value of more complex models differed depending on how value was assessed. Although the most complex model, based on detailed movement behaviors, best predicted observed dispersal rates, it was only slightly better than the simplest model, which was based solely on distance between sites. Consequently, a parsimony approach using information criteria favors the simplest model we examined. However, when we applied the models to a larger landscape that included proposed habitat restoration sites, in which the composition of the matrix was different than the matrix surrounding extant breeding sites, the simplest model failed to identify a potentially important dispersal barrier, open habitat that butterflies rarely enter, which may completely isolate some of the proposed restoration sites from other breeding sites. Finally, we found that, although the gain in predicting dispersal with increasing model complexity was small, so was the increase in financial cost. Furthermore, a greater fit continued to accrue with greater financial cost, and more complex models made substantially different predictions than simple models when applied to a novel landscape in which butterflies are to be reintroduced to bolster their populations. This suggests that more complex models might be justifiable on financial grounds. Our results caution against a pure parsimony approach to deciding how complex movement models need to be to accurately predict dispersal through the matrix, especially if the models are to be applied to novel or modified landscapes.
\end{abstract}

Key words: capture-mark-recapture; connectivity; dispersal; habitat fragmentation; matrix habitat; Neonympha mitchellii francisci; restoration; spatially explicit individual-based simulation model.

\section{INTRODUCTION}

Ecologists and conservation biologists now widely appreciate the potential importance of dispersal for the persistence of species in fragmented landscapes (Gonzalez et al. 1998, Hanski 1998, Crooks and Sanjayan 2006, Beier et al. 2008). First-generation tools to infer dispersal (e.g., incidence function models; Hanski 1994) assumed that the landscape between two habitat patches (the "matrix") was homogeneous, or that the probability of successful dispersal depended only on the summed distances of all paths linking the patches (McRae and Beier 2007). However, more recent studies

Manuscript received 9 June 2011; revised 2 March 2012; accepted 13 March 2012. Corresponding Editor: C. Gratton.

6E-mail: hudgens@iws.org have demonstrated that dispersal often differs between different types of matrix habitat, altering the "effective distance" between patches (Ricketts 2001, Revilla et al. 2004, Revilla and Wiegand 2008). Moreover, individual dispersers may alter their trajectories in response to boundaries between different types of matrix habitat that they encounter as they cross the landscape (Schultz and Crone 2001, Levey et al. 2005, Kuefler et al. 2010).

Because habitat-specific movement and boundary behaviors will change the effective distance between suitable habitat patches, distance alone may be a poor indicator of inter-patch movements. Nonetheless, whereas inter-patch distances are easily obtained from a map or a GIS database, it is more costly in time and financial resources to measure among-habitat differences in movement and boundary behaviors, and to 
incorporate them into spatially explicit models. Because of this, landscape resistance is in practice typically determined by expert opinion (Beier et al. 2008, 2009). Even when movement parameters are estimated from data, dispersal predictions may be particularly sensitive to errors in those estimates (Ruckelshaus et al. 1997). For all of these reasons, a key question arises: how much complexity, in both movement behavior and the matrix landscape, must we incorporate into models to accurately predict dispersal of individuals between patches of breeding habitat?

We address this question using wetland butterflies as a model system. Our motivating species is the St. Francis' satyr butterfly (Neonympha mitchellii francisci, hereafter SFS), a U.S. federally endangered species found only at Ft. Bragg, North Carolina, a U.S. military base. It occurs only in a small number of sedge-dominated wetlands along headwater streams. Because this butterfly is so rare, it can be difficult to study its dispersal. Thus, we have measured habitat-specific movements and boundary behaviors for the closely related Appalachian brown butterfly (Satyrodes appalachia) within SFS breeding and non-breeding (i.e., matrix) habitats (Kuefler et al. 2010), and we use Appalachian brown as a surrogate species here. We quantified Appalachian brown movements in all common natural and developed habitats and at all possible habitat boundaries. We then used these movement data to construct simulation models of butterfly dispersal that we ran on a digital representation of the landscape the butterflies occupy at Ft. Bragg. Specifically, we constructed a range of models of increasing complexity, from the simplest model in which movements of butterflies are the same in all habitats and they ignore boundaries (equivalent to a distance-only model) to the most complex model that includes habitat-specific movements and boundary behaviors. We then compared our simulation results to SFS dispersal data collected independently though capture-mark-recapture (CMR) studies involving all known, easily accessible SFS sites (Haddad et al. 2008).

As we noted above, building a more complex model involves greater cost. Therefore, even if a more complex model does a better job of predicting dispersal than does a simpler model, the simpler model might be preferred if it is easier to build and parameterize but still does a reasonable job predicting dispersal. We assume that more complex models require additional information to build and parameterize than simpler models. Hence our approach considers the value of information for management decisions (McCarthy and Possingham 2007, Martin et al. 2009). We assessed the value of building more complex models in three ways. First, we adopted a parsimony approach using information criteria, weighing overall ability to predict observed dispersal against the number of parameters that needed to be estimated to run the model. Second, we compared the predictive ability of the models to the financial costs of estimating the parameters.
The third way we assessed the value of model complexity considers potential applications to landscape management. Assessing a model's ability to predict observed dispersal among existing SFS populations is an incomplete test of that model's usefulness for predicting dispersal on a landscape altered by management. Pairs of potential source and destination sites in our CMR study were separated by either short distances of wetland matrix or by very large distances that SFS would not be expected to traverse regardless of matrix composition. Therefore, including details of movement differences in multiple habitats and at different boundary types in the model may have been unnecessary to predict the CMR results. However, as is the case for SFS, an important goal in constructing dispersal models is to provide a tool for evaluating the consequences of management actions that may create a landscape that differs from the one on which the model was validated. Efforts are now under way to create (through flooding and tree removal) restored wetlands near current breeding sites and to translocate SFS into them. Some of these restoration sites will be located closer to habitat types that butterflies encounter only rarely in the vicinity of current breeding sites. The movement models we have constructed are intended to aid in assessing whether proposed restoration sites are likely to be connected by dispersal to other breeding sites (both current and restored). Therefore, we also ask: would using one model vs. another recommend different actions? Specifically, would the different models lead one to select different locations to be restored?

\section{Methods}

St. Francis' satyr is limited to at most 50 ha of wetland habitat at Ft. Bragg, North Carolina (see Plate 1). It is restricted primarily to abandoned beaver ponds, where early successional vegetation is dominated by sedges (Carex spp.), the butterfly's likely host plants. Local extinction is inevitable, because late successional habitat is unsuitable and butterflies cannot survive flooding of wetlands by beavers. Beavers were driven to extinction in North Carolina by 1897, but have since recovered. It is likely that SFS persisted at Ft. Bragg in the absence of beavers because of army activities, as the population is concentrated within artillery impact areas. In those areas, artillery and flares ignite fires almost every year, and in dry years the fires burn through wetlands and retard succession.

\section{Movement and landscape parameters}

To parameterize spatially explicit dispersal simulation models, we observed movement behaviors for butterflies in their native wetland habitat and in release experiments conducted in three other common habitat types in our landscape, riparian forest, upland longleaf pine forest, and open habitats (grasslands or developed areas). Butterflies were released in and at the edges between all habitat types and tracked for up to 30 
minutes. Movement rates from release experiments were validated against movement rates for butterflies in native wetlands that had never been captured. The details of the data collection are presented elsewhere (Kuefler et al. 2010).

Our movement experiments involved displacing individuals from their native wetlands to other habitats, and we could not be certain that we would be able to recapture all released butterflies to return them to wetlands. Thus, we judged that it would be unethical to perform those experiments on the endangered SFS, and we conducted our studies on a surrogate species, the Appalachian brown butterfly (Satyrodes appalachia). We made this decision based on (1) the high degree of overlap in the habitat distributions of these two species at Ft. Bragg (N. Haddad, personal observation); (2) their close phylogentic relatedness; (3) their similarity in host plant use; and (4) similar movement behaviors exhibited by naturally moving (non-released) individuals of the two species in wetlands. Specifically, the distributions of turn angles for the two species were indistinguishable, as were the fractions of butterflies approaching wetlandupland boundaries that crossed into upland habitat. Appalachian browns differed from SFS in that they had longer step lengths on average (Appendix A).

Two types of movement data, obtained by recording the positions of butterflies at 5-s time intervals, were used to estimate model parameters. First, the propensity for a butterfly to move was represented by (1) the probability that a moving butterfly continued to move in the following time interval; and (2) the time that a resting butterfly remained at rest before initiating its next move. Second, butterfly behavior while moving was represented by (1) the distribution of the distance moved during a time interval (step lengths); (2) the magnitude of directional change between consecutive intervals (turn angles); and (3) the probability that a butterfly on the edge between two habitats entered one habitat and not the other. Except as noted in Types of simulations and scenarios simulated, different step length and turn angle distributions were used for each habitat.

The dispersal models required data on the spatial arrangement of habitats at Ft. Bragg, which we obtained from the 2001 National Landcover Database (Homer et al. 2004). These data were scaled to $30 \times 30 \mathrm{~m}$ cells. We also obtained data on the locations of breeding wetlands for SFS. These data included a layer of sites maintained by Ft. Bragg, observations of where SFS bred that we recorded during annual population surveys (Kuefler et al. 2008), proposed restoration sites at Ft. Bragg, and suitable sites as determined by Maxent niche modeling (Phillips et al. 2006). The niche model was based on 217 SFS occurrences, and on landscape information that included (1) terrain data derived from the National Elevation Dataset (Gesch et al. 2002); (2) land cover data derived from cloud- and snow-free LANDSAT-5 Thematic Mapper (TM) images, one taken in winter (January 2008) and one in summer (May 2008); and (3) vegetation structure data obtained by flying an airborne Optech ALTM 2050 LiDAR System over Ft. Bragg in early July 2006. We distinguished suitable from unsuitable habitat using the sensitivity-specificity equality approach (Liu et al. 2005).

\section{Spatially explicit individual-based simulations}

We simulated butterfly movement using individualbased models coded in MATLAB 7.0 (The MathWorks, Natick, Massachusetts, USA). Each simulation began with a single butterfly placed in the center of each cell located within known breeding sites and assigned a random starting direction. At the beginning of the first time step, each butterfly either rested, with probability $p_{\mathrm{w}}$, the resting probability in wetlands, or moved, with probability $1-p_{\mathrm{w}}$. If the butterfly rested, the number of time steps before the next move was drawn from the distribution of observed rest times, and the butterfly's location remained the same for that period. If the butterfly moved, a move distance was drawn from a gamma distribution with wetland-specific parameters and a turn angle was drawn from a smoothed frequency distribution fit to observed data from wetland releases. We ruled out fitting unimodal probability distributions for turn angles (cf. Shultz and Crone 2001) because the empirical distribution of turn angles in riparian habitats was strongly bimodal. Smoothing was accomplished by first drawing a random 30 degree bin with probability equal to the observed frequency of turns in each range, and then drawing a turn angle within that range from a uniform distribution. Finally, the turn was assigned to be to the right or left with equal probability. The resulting turn angle determined the new direction of movement, and the step length then determined the possible new position. If the new position was within the same cell, or the new cell and all intervening cells were the same habitat as the original cell, the move was allowed and the butterfly's location was assigned to be the new position.

If, however, the new position resulted in the butterfly crossing a boundary to a different habitat, the program then determined if the move was allowed. In our experimental releases, we quantified the fraction $F$ of butterflies starting at rest at the boundary between two habitats that moved into the more preferred habitat. To parameterize the simulation models, we had to translate $F$ into the probabilities that butterflies would cross boundaries when approaching from either the more- or the less-preferred habitat. To do so, we first assumed that all butterflies approaching the more preferred habitat would cross the boundary. If we further assume that our releases correspond to half of the butterflies encountering the boundary from the more-preferred and half from the less-preferred habitat, then

$$
F=\left(\frac{1}{2} \times 1\right)\left(\frac{1}{2} \times P\right)
$$

where $P$ represents the probability that a butterfly 
approaching the boundary from within the more preferred habitat remains in that habitat. Thus we estimate $P$ as $2(F-[1 / 2])$, and the probability that a butterfly moves from more- to less-preferred habitat is 1 $-P$.

If the move into less preferred habitat was allowed, the butterfly's location was updated. If not, a new step length and turn angle were drawn, and the process repeated until either the new location was in the original cell or the boundary was crossed. If a new location would take a butterfly across multiple cells in a single step, all boundary crossings had to be allowed.

This process was repeated using the resting probability, rest time distribution, step length distribution, and turn angle distribution from the habitat corresponding to the butterfly's current location until the butterfly died. We found no detectable difference in rest times among habitats, so we used the pooled data to create a single distribution for all habitat types. Each butterfly was assigned a lifespan drawn from an exponential distribution with a mean of 3 days, which provided the most parsimonious fit to the CMR data (Kuefler et al. 2008). Each day was composed of 4320 5-second time steps covering the 6-hour period when SFS were observed to be active during daily monitoring of breeding sites (N. M. Haddad and B. R. Hudgens, personal observations). We had no data on habitat specific mortality and thus assumed that it did not differ among habitats.

\section{Types of simulations and scenarios simulated}

The model described above (hereafter the "full simulation") represented the most complex model we used. To evaluate the need for such a complex model, we compared its ability to predict observed dispersal with that of three simpler models. In the no boundary behavior model, we retained habitat-specific movements (i.e., distributions of turn angles, step lengths, and rest durations) but eliminated boundary behaviors (so that simulated butterflies moved freely from one habitat to another). In contrast, in the boundary behavior only model, we applied the movement characteristics from the breeding habitat (wetlands) to all habitat types, but we retained boundary behaviors where different habitats meet. Finally, in the distance only model, we applied wetland movement characteristics to the entire landscape with no boundary behaviors (thus simulating a pure correlated random walk, in which dispersal should depend only on the distance between sites). We used only wetland data because it represents the simplest scenario of quantifying movement behavior only where the butterflies are most often found; quantifying behavior in other habitats requires experimental releases, as done by Kuefler et al. (2010).

To determine the movement detail that is needed to accurately represent dispersal, we compare dispersal predicted in simulations of varying complexity to dispersal events observed in a capture-mark-recapture (CMR) study of SFS marked in multiple breeding sites in 5 years (Haddad et al. 2008). Importantly, the dispersal data we used in the assessment are independent of the movement data we used to estimate model parameters.

Briefly, we uniquely marked and recaptured butterflies through approximately daily surveys of all known and accessible breeding sites during the second flight period of each year from 2003-2007, and in the first flight period of 2005 (details provided in Haddad et al. 2008). Three of the larger sites were subdivided into sub-sites. In addition, we informally surveyed for butterflies flying in upland habitat adjacent to the three subdivided sites in locations used as staging areas. We estimate that approximately half as much effort was spent searching for butterflies in each of the three upland sites as was spent surveying breeding habitats. For the current study, we include all movements either within or between populations or between populations and adjacent upland sites. Altogether, we recaptured 403 butterflies, of which 102 had moved to a different site.

For all models, we simulated two scenarios. The first scenario included only the existing breeding sites and the landscape surrounding them. As some new breeding sites appeared and were added to the study while other sites went extinct, we updated the simulated landscape each year of the CMR study and compared observed and predicted dispersal events on a year-by-year basis.

In the second scenario, we added proposed restoration sites to the landscape that includes the existing breeding sites, and we assumed new populations had successfully established in these sites. The proposed restoration sites were initially chosen based on their potential to be converted to suitable habitat. This second scenario represents a real-world application of our model: restoration is currently being performed at a subset of the proposed sites. This scenario has the additional benefit of adding complexity to our landscape that areas within the dispersal distance of existing SFS populations lack. The landscape immediately surrounding existing breeding sites has very little open habitat. Behaviors in open habitat differed most from all other habitats: butterflies were much less likely to enter open habitat, but, once there, they moved more rapidly (Kuefler et al. 2010). Because there was no open habitat near current butterfly sites, incorporating behaviors we have estimated in open habitat and at boundaries between open and the other types of habitat into the more complex models did little to improve their fit to the CMR data relative to the distance only model (see Results). However, establishing new SFS breeding sites in restored wetlands would put some butterflies in closer proximity to open habitat. Assuming butterflies in restored sites would exhibit the same movement behaviors as butterflies in natural, unrestored wetlands, we used the four movement models to simulate dispersal of butterflies between all breeding sites (natural and restored), and we compare those predictions among models. 

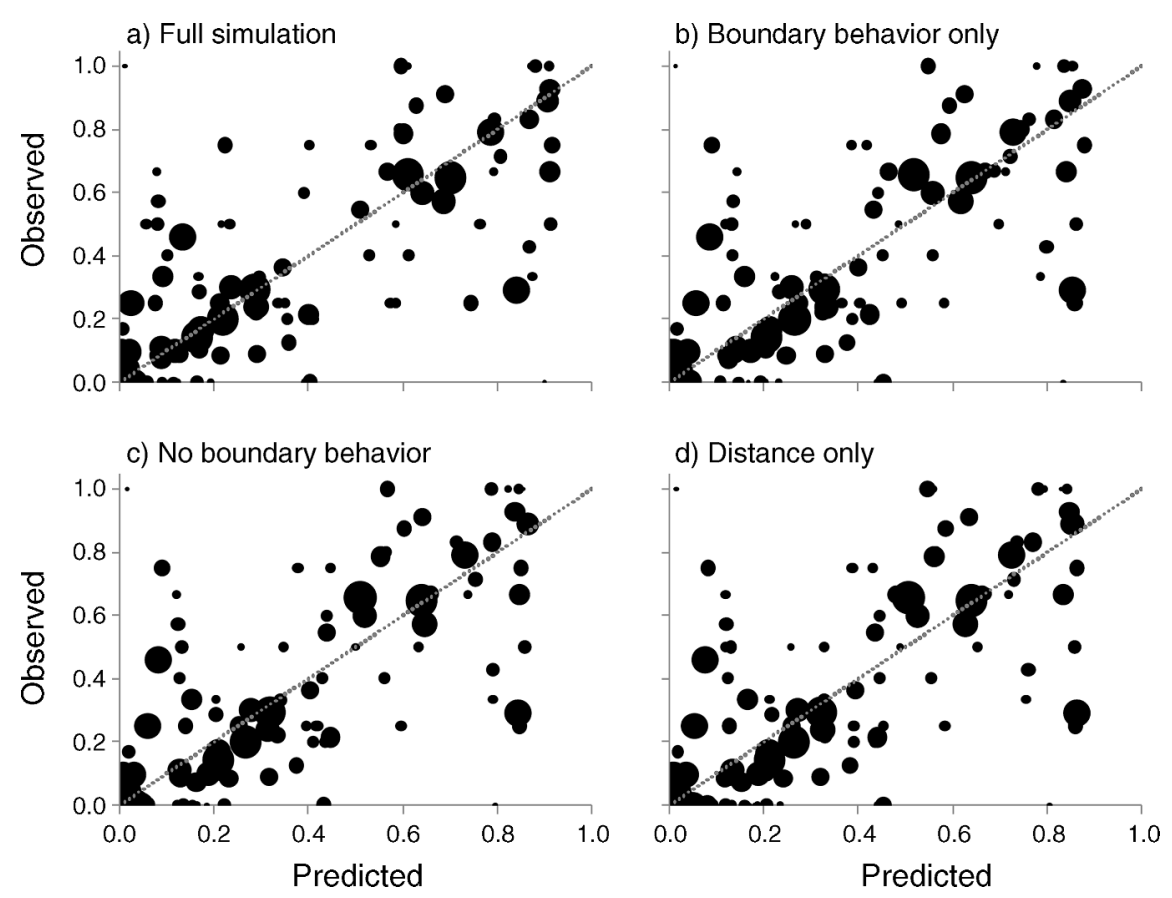

FIG. 1. Predicted and observed dispersal rates between paired survey sites in the capture-mark-recapture study (all years combined). Each point represents the fraction of simulated and observed marked St. Francis' satyr butterfly (Neonympha mitchellii francisci; SFS) dispersing between a pair of surveyed sites, including SFS resighted in the same site where they were originally marked, during a single flight period. The size of the point indicates the number of recaptured SFS originating from a breeding site (range 1-35).

\section{Statistical analyses}

We assessed the fit between model predictions based on Appalachian brown butterfly movement behaviors and observed SFS dispersal events in the CMR study in two ways. First, we computed the correlation between the observed fractions of dispersers starting from each breeding site recaptured at all surveyed sites (including the site of origin) and the fractions predicted by each model. To account for differences among breeding sites in the number of recaptured butterflies, we computed weighted correlations, and we used a nonparametric bootstrap to place $95 \%$ confidence intervals on the weighted correlations using the adjusted bootstrap percentile (BCa) method (R Development Core Team 2005). This approach does not discount for the number of parameters used in the model. Second, we computed the multinomial likelihoods (Appendix B) of the observed recaptures for each model, and used them to compute the sample-size-corrected Akaike's information criterion (Hurvich and Tsai 1989), which penalizes models with more parameters (see Appendix B for a description of how we determined the number of parameters in each model).

We compared model fit to the marginal cost of estimating additional parameters in more complex models. The background cost of performing the CMR study would have had to be paid to validate any of the models, or even to test predictions made in the absence of movement data, such as those based on simple map distance, incidence function models, or expert opinion. Beyond this background cost, the simplest (i.e., distance only) model required the additional (marginal) cost of measuring movement behaviors in breeding (i.e., wetland) habitat, and parameterizing the three more complex models required the additional (marginal) cost of measuring movement in multiple habitats (no boundary behavior model), behavior at habitat boundaries (boundary behavior only model), or both (full simulation model). Details of these costs are provided in Appendix B.

\section{RESULTS}

\section{Model validation using capture-mark-recapture data}

For all four movement models, the predicted probabilities of dispersal among sites within the same drainage were significantly, positively correlated with dispersal observed in the CMR study (Fig.1, Table 1). As predicted in our models, no marked butterfly moved between drainages. The estimated correlation between observed and predicted dispersal was highest for the full model (the most complex model) and lowest for the distance only model (the least complex model). However, the three simplest models produced correlations that were only very slightly lower than, and fell well within the $95 \%$ confidence interval for, the correlation produced by the full model. Similarly, the log likelihood was highest for the full model and lowest for the distance only model, but the likelihoods of the three simpler 
TABLE 1. Measures of fit and cost for simulation models of differing complexity.

\begin{tabular}{lcccc}
\hline \hline & \multicolumn{4}{c}{ Dispersal simulation model } \\
\cline { 2 - 5 } & Full simulation & No boundary behavior & Boundary behavior only & Distance only \\
\hline Correlation & $0.832(0.795,0.877)$ & $0.825(0.790,0.873)$ & $0.820(0.781,0.869)$ & $0.820(0.783,0.868)$ \\
Log likelihood & -357.445 & -361.027 & -359.621 & -364.643 \\
Number of parameters & 44 & 32 & 20 & 8 \\
AIC & 813.98 & 791.78 & 761.45 & 745.65 \\
$\Delta$ AIC $_{\mathrm{c}}$ & 68.33 & 46.13 & 15.80 & 0 \\
Marginal costs (\$U.S.) & 10600 & 5800 & 7000 & 2200 \\
\hline
\end{tabular}

Notes: "Correlation" gives the weighted Pearson correlation coefficients (with $95 \%$ confidence intervals in parentheses) between the observed fractions of recaptured St. Francis' satyr butterfly (Neonympha mitchellii francisci, SFS) moving between specific pairs of origin and destination sites in the capture-mark-recapture (CMR) study and the probabilities of dispersal between those site pairs predicted by the four dispersal models. Correlations are weighted by the number of butterflies recaptured from each breeding site; each confidence interval is based on 4999 bootstrap samples. Log likelihood is for a multinomial model (see Appendix B). $\Delta \mathrm{AIC}_{\mathrm{c}}$ is the difference in $\mathrm{AIC}_{\mathrm{c}}$ value from the model (i.e., distance only) with the lowest value.

models were only slightly lower than that of the full model. Discounting these log likelihoods for the number of parameters in each model, the distance only model emerges with the lowest $\mathrm{AIC}_{\mathrm{c}}$ value. Thus, a strict parsimony approach based on the available CMR data favors the simplest model.

\section{Comparison of model predictions on a landscape that includes proposed restoration sites}

As observed in the landscape without restored wetlands (maps not shown), the simulations with restored wetlands predict little or no dispersal between habitat patches separated by more than a few hundred meters, such that SFS is predicted to occupy a collection of isolated networks containing one or a few nearby breeding sites (Fig. 2). However, at a smaller scale, predicted dispersal distances and routes differ among models. The simplest (distance only) model predicts the farthest distance traveled from breeding sites, with dispersal paths extending out $480 \mathrm{~m}$. Dispersal patterns predicted by the boundary behavior only model are generally similar to those of the distance only model. In contrast, dispersal paths in the full simulation extend at most $360 \mathrm{~m}$ from sources. The no boundary behavior model predicts dispersal patterns similar to those of the full simulation.

Models differ in predicted dispersal rates between established SFS populations and nearby proposed restoration sites. The distance only model predicts relatively high dispersal among proposed restoration sites and that most of these sites will be linked to established SFS populations, while the full simulation predicts relatively low dispersal among proposed restoration sites, with three sites completely isolated from any other breeding site and no dispersal between restored and currently occupied sites (Fig. 2). Incorporating habitat effects on within-habitat movement (as in the no boundary behavior model and the full simulation) leads to the greatest reduction in dispersal among most sites, while boundary behaviors act to isolate sites separated by open areas or roads (Fig.2; sites r11 and r12).
Two proposed restoration sites (Fig. 2; sites r1and r2) are predicted by all models to be connected to existing breeding colonies, and sites in two additional site clusters (Fig. 2; sites r3-r4 and the cluster of four sites r5-r8) are predicted by all models to be connected to other restored sites within the same cluster. These eight sites ( $\mathrm{r} 1-\mathrm{r} 8)$ were chosen as restoration sites and are currently being restored.

\section{Model complexity vs. marginal cost of parameter estimation}

As the simulations on the landscape with restoration sites show that more complex models may indicate barriers to dispersal that simpler models miss (Fig. 2), it is worthwhile to assess the financial cost effectiveness of increasing model complexity, which we did by comparing the ability of the models to predict the CMR results to the marginal cost of estimating model parameters (Fig. 3). Even though the gain in fit (measured by either the correlation or the log likelihood) is small, the marginal cost of achieving that gain is also relatively modest. Moreover, there is no sign of diminishing returns, as would be indicated by a plateau in fit with increasing marginal cost (although with only four points, one per model, we cannot evaluate this claim statistically). Importantly, because we can only assess model fit on the landscape without restoration sites on which the CMR results were obtained, the cost effectiveness of constructing more complex models may be higher than this analysis indicates. This is especially so if it turns out that the more complex models accurately predict that open habitat will pose a barrier to dispersal between restored and current breeding sites, which can only be evaluated after the restoration has been completed.

\section{DiscusSION}

Our answer to the question "how valuable is it to construct more complex models of dispersal through matrix habitats?" differed depending on how value was assessed. From a strict parsimony viewpoint, the (at best) small increase in the ability of more complex 
Full simulation

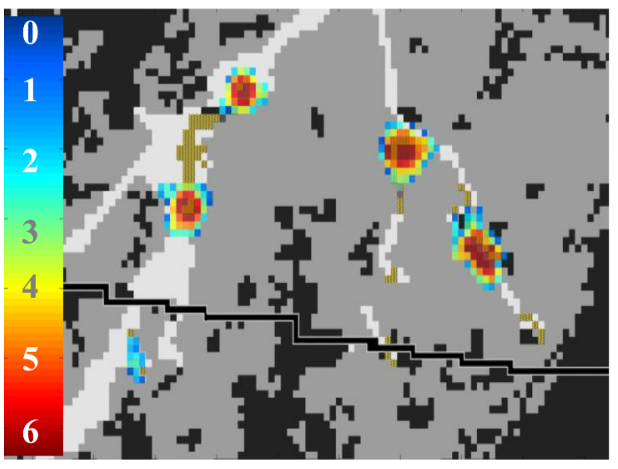

No boundary behavior

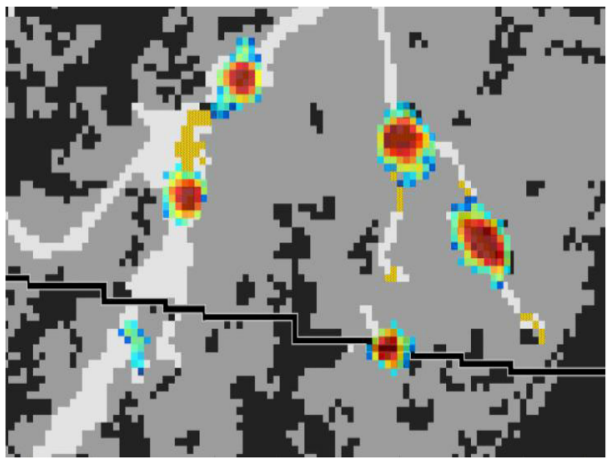

Breeding sites

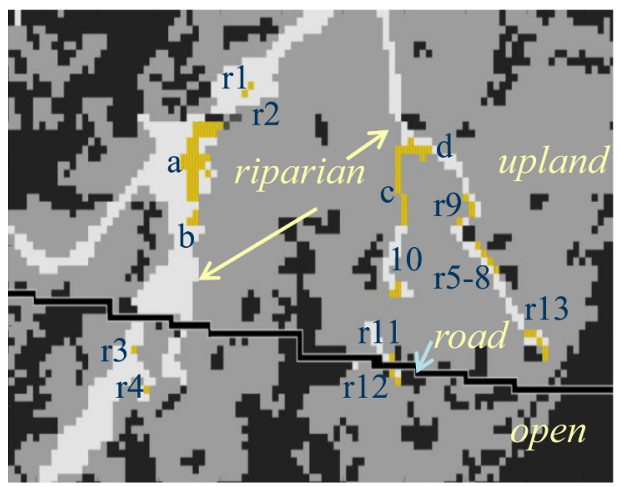

Boundary behavior only

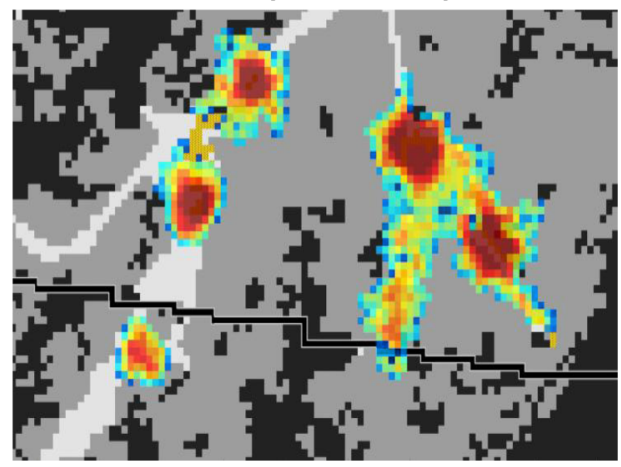

Distance only

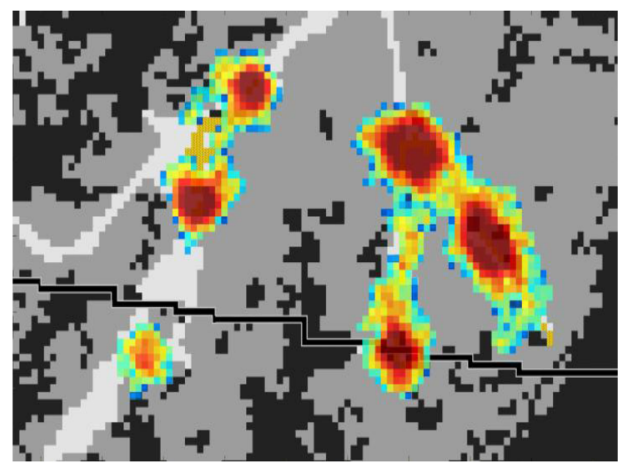

(Full simulation) - (Distance only)

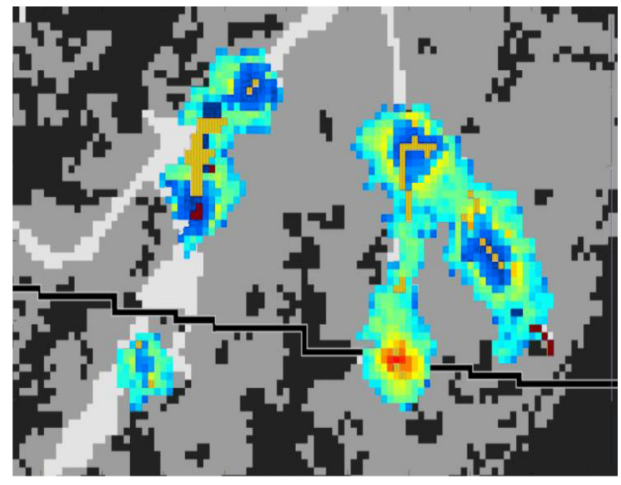

FIG. 2. Predicted butterfly dispersal patterns after habitat restoration. The top four panels show heat maps indicating the relative frequency with which a cell was crossed during simulated dispersal events among existing breeding sites, among restored sites, or between existing and restored sites. Warmer (redder) colors indicate more frequent use. The scale bar indicates the natural logarithm of the number of dispersal paths crossing a pixel indicated by the corresponding colors. Areas in any shade of gray were not used by simulated butterflies. Different shades of gray indicate the habitat type of the cell: riparian corridor (lightest hue), upland forest (next darkest hue), and open habitats (darkest hue). The bottom left panel shows a map of the area targeted for restoration and the location of habitats. Breeding sites are shown in gold with extant breeding sites labeled a-d and clusters of proposed restoration sites labeled $\mathrm{r} 1-\mathrm{r} 13$. Breeding sites $\mathrm{c}$ and $\mathrm{d}$ represent two sub-sites within a single occupied site to illustrate the scale of within-site dispersal as measured in the mark-recapture study. Except for r9, r10, and r13, restoration sites are $30 \times 30 \mathrm{~m}$, corresponding to the pixel size in the figure and coarseness of the habitat map. The bottom right panel shows the difference in the number of times a cell was used during simulated dispersal events between the distance only and full simulation models.

models to predict the CMR results was negated by the substantial increase in the number of parameters that had to be estimated before those models could be used. Thus an information criterion approach using the CMR data strongly favors the simplest model we examined. However, when we applied the models to a larger, post- restoration landscape, our results caution that the simplest model may fail to identify important barriers to dispersal, specifically open habitat that may isolate some of the proposed restoration sites from other breeding sites (Fig. 2). Finally, we found that, although the gain in fit to the CMR data with increasing 


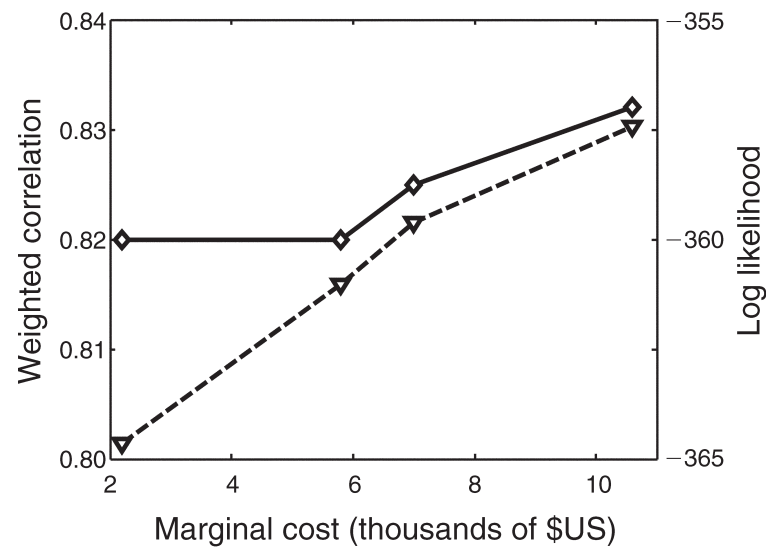

FIG. 3. Measures of model fit vs. the marginal cost of collecting more data to parameterize more complex models. The solid line shows the weighted correlation, and the dashed line shows the log likelihood.

complexity was small, so was the increase in financial cost. Greater fit continued to accrue with greater financial cost, suggesting that constructing more complex models might be justifiable on financial grounds. It is important to note that we needed the CMR data, which incurred significant cost in itself, to validate our models. Yet we were able to predict dispersal well with cheaper-to-collect data on movement behaviors. In the following paragraphs, we discuss the apparent conflicts between these different views of the conservation value of more complex movement models.

The mismatch we found between different ways of evaluating complex models is best understood in terms of the conflict between what is practical and what is desirable when studying and managing rare species. We conducted our CMR study using every known, easily accessible breeding site for SFS. Because SFS dispersal out of its breeding habitat (wetlands) is extremely limited, and its current distribution is highly restricted, the marked butterflies in the CMR study only "sampled" a limited proportion of the landscape very close to existing breeding sites. That region has low representation of open habitats such as fields and roads. It would be impractical to do the CMR study anywhere but where we did, but it is desirable to know how butterflies will respond to novel environments. Movement of the surrogate species (Appalachian browns) was very different in open habitat than in any other habitat, and individuals were reluctant to move into the open when released at the boundary between open and other habitats (Kuefler et al. 2010). Nevertheless, those details are irrelevant if butterflies rarely encounter open habitat, as was the case for the marked SFS butterflies in the CMR study. Under these circumstances, adding complexity to the model by allowing it to account for the consequences of rare encounters with open habitat is difficult to justify solely on the basis of a model's ability to predict the CMR results in a parsimonious way.
However, as with many other rare species, establishing new breeding sites through restoration is a desirable management goal. For SFS, the most suitable locations for restored wetlands would put butterflies in closer proximity to habitat types, notably open habitat, that are rarer in the vicinity of existing breeding sites. Moreover, several of the proposed restoration sites are completely separated from existing sites by a strip of open habitat (a road; Fig. 2). For species reduced to a very small fraction of their historic range (Schultz and Crone 2001) or to areas where widespread habitat conversion has modified the matrix surrounding remnant sites (Kolozsvary and Swihart 1999), it is likely that the efficacy of habitat restoration will depend on how well individuals disperse through landscapes that differ strongly from those where the species can be studied naturally.

Our results suggest that it may be dangerous to base decisions about which potential restoration sites to use solely on simpler movement models, even if they explain more parsimoniously dispersal patterns within less diverse landscapes. In particular, more complex models that incorporate documented responses of butterflies to open habitat predict that restoration sites separated from existing breeding sites by open habitat may experience only very rare exchange of dispersers with existing sites (Fig. 2; sites $\mathrm{r} 10$ and r11). Although proposed sites $\mathrm{r} 10$ and $\mathrm{r} 11$ were removed from the portfolio for initial restoration based on other considerations (i.e., their restoration potential), the complex model (but not the simple model) supported that decision on the basis of connectivity concerns. If those sites are to be restored in the future, the complex (but not simple) model would recommend that additional management, such as removing the road as a barrier, might be needed to ensure connectivity. More generally, complex movement models may help to identify landscape-scale elements that might block dispersal into restoration sites, threatening the success of conservation efforts (George and Zack 2001).

A caveat is that the complex model has not been tested for SFS within a landscape that includes open habitats. Because some details of movement behaviors differ between SFS and Appalachian brown butterflies, predictions of dispersal patterns in restoration landscapes should be interpreted with caution. For example, differences in step lengths between the species (Appendix A) may be exacerbated in open habitats, where the step lengths may be most sensitive to differences in the butterflies' physiology or morphology (Appalachian brown butterflies are slightly larger than SFS). However, the primary impact of open habitat on butterfly dispersal is not driven by movement behavior in the open, but by a strong aversion to entering open habitat, a behavior shared by both butterfly species (Kuefler et al. 2010). Nonetheless, decisions about where to locate SFS restoration sites based on our model should consider the possibility that SFS and Appalachian 

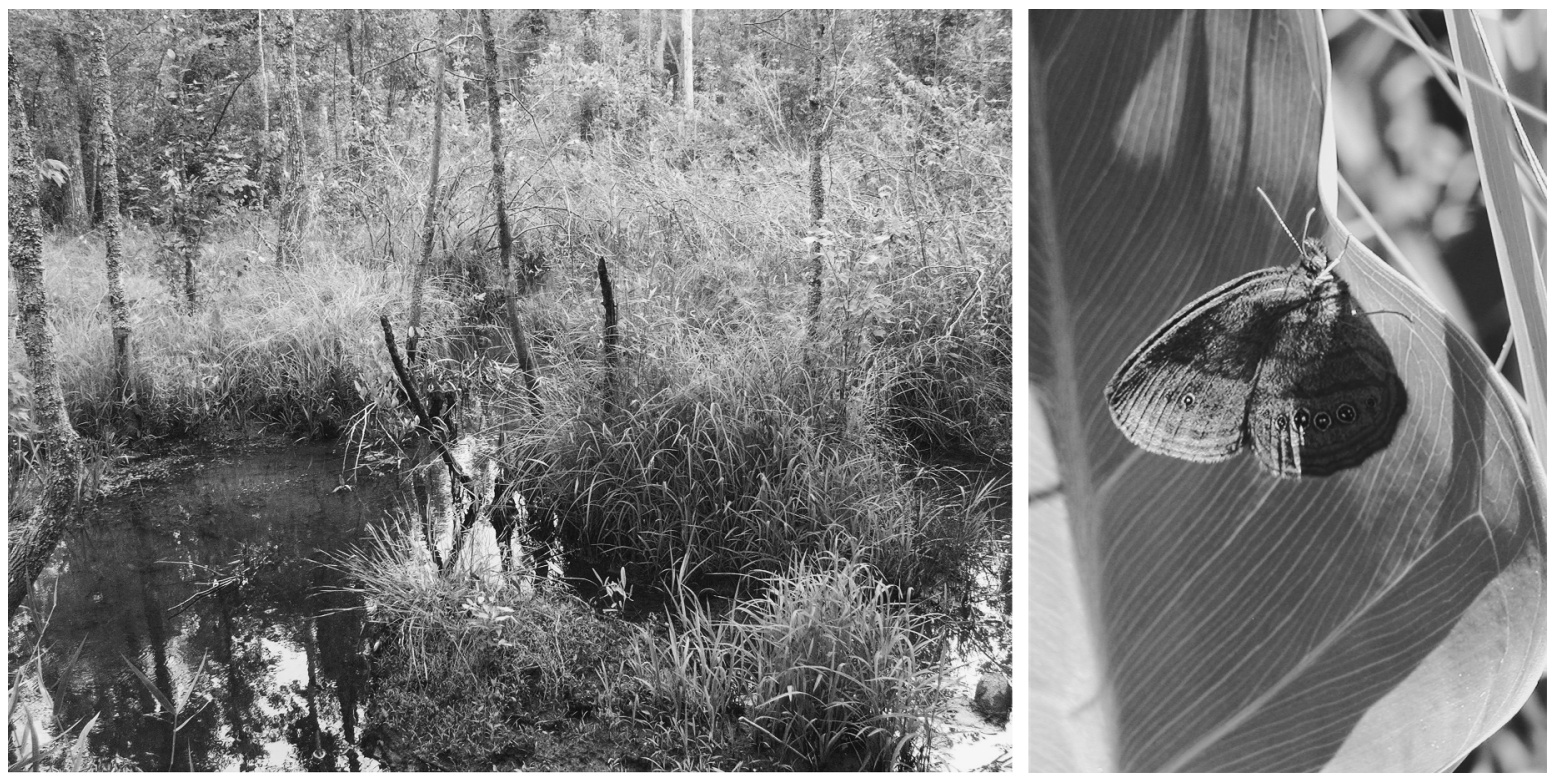

Plate 1. Dispersal of (right) Saint Francis' Satyrs (Neonympha mitchellii francisci) depends on movement behaviors in (left, foreground) wetland habitats where they breed, and (left background) surrounding upland forest or riparian corridors, and at the edges where habitats meet. Photo credits: B. R. Hudgens.

brown butterflies may respond differently to open habitats, and SFS releases into restored sites should be done in a way that facilitates tests of model predictions (e.g., testing whether dispersal occurs more frequently between pairs of restored sites not separated by roads).

There are at least two additional complexities that we did not include in our models but may have important impacts on model predictions. First, we assumed that butterfly survival is not influenced by matrix habitat type. Although differential survival between habitat types is a strong predictor of how matrix habitats influence dispersal rates (Hudgens and Haddad 2003), this parameter is particularly difficult to estimate. We also assumed that movement behaviors do not differ between natural and restored habitats, an assumption that can only be tested once the new restoration sites have been established. Once restoration has occurred, a future CMR study in the restored landscape with a different representation of matrix habitats relative to the landscape on which the original CMR study was conducted could provide new information to assess the value of simple vs. complex movement models.

Our simulations based only on behavioral data did a remarkably good job of predicting the dispersal patterns we documented in the CMR study (Fig. 1). If our model had not required validation, these behavioral data alone would have been much cheaper to collect than the CMR data, and the marginal cost of gathering additional behavioral data for the more complex models would be relatively low. Although we have not done so, we could assess whether even lower marginal costs might be achieved, by removing individual butterflies from our data set and assessing whether a smaller (and thus cheaper) study would still have provided sufficient power to detect habitat differences in behavior, or to accurately predict the CMR results. The success of our models offers hope that movement studies integrated with spatially explicit individual-based simulations may provide more cost-effective tools for assessing landscape connectivity than do CMR methods, at least for species for which it is feasible to conduct movement observations. Moreover, because they are more mechanistic, such simulations have the advantage that they can be applied to novel landscapes, as we have advocated here. Partitioning dispersal paths into movement behavior within different habitat types and movement behavior at habitat boundaries provides a viable approach for linking small-scale behaviors to landscape-level processes (Lima and Zollner 1996, Levey et al. 2005, Caro 2007, Leidner and Haddad 2011, Wang et al. 2011).

There is no single level of model complexity that will work for all circumstances. The optimal complexity depends on the degree of matrix heterogeneity and future modification of the landscape, as well as the marginal cost of additional data. Marginal costs will vary from study to study, although the approach we have adopted here of assessing the increase in a model's predictive ability against the increase in marginal costs (Fig. 3) is generally applicable. Our study highlights how understanding the landscape-level behavioral ecology of a focal species can provide effective and efficient means to identify strategies for its conservation and restoration.

\section{ACKNOWLEDGMENTS}

We thank the Strategic Environmental Research and Development Program and the Ft. Bragg Endangered Species Branch for financial support, Brian Ball and Erich Hoffman for 
assistance in the field, and Aaron Moody, Jeff Walters, and Anne Trainor for comments and suggestions on the work.

\section{Literature Cited}

Beier, P., D. R. Majka, and S. L. Newell. 2009. Uncertainty analysis of least-cost modeling for designing wildlife linkages. Ecological Applications 19:2067-2077.

Beier, P., D. R. Majka, and W. D. Spencer. 2008. Forks in the road: choices in procedures for designing wildland linkages. Conservation Biology 22:836-851.

Caro, T. 2007. Behavior and conservation: a bridge too far? Trends in Ecology and Evolution 22:394-400.

Crooks, K. R., and M. A. Sanjayan. 2006. Connectivity conservation. Cambridge University Press, Cambridge, UK

George, T. L., and S. Zack. 2001. Spatial and temporal considerations in restoring habitat for wildlife. Restoration Ecology 9:272-279.

Gesch, D., M. Oimoen, S. Greenlee, C. Nelson, M. Steuck, and D. Tyler. 2002. The National Elevational Dataset. Photogrammetric Engineering and Remote Sensing 68:5-11.

Gonzalez, A., J. H. Lawton, F. S. Gilbert, T. M. Blackburn, and I. Evans-Freke. 1998. Metapopulation dynamics, abundance, and distribution in a microecosystem. Science 281:2045-2047.

Haddad, N. M., B. Hudgens, C. Damiani, K. Gross, D. Kuefler, and K. Pollock. 2008. Determining optimal population monitoring for rare butterflies. Conservation Biology 22:929-940.

Hanski, I. 1994. A practical model of metapopulation dynamics. Journal of Animal Ecology 63:151-162.

Hanski, I. 1998. Metapopulation ecology. Oxford University Press, Oxford, UK.

Homer, C., C. Huang, L. Yang, B. Wylie, and M. Coan. 2004. Development of a 2001 National Landcover Database for the United States. Photogrammetric Engineering and Remote Sensing 70:829-840.

Hudgens, B. R., and N. M. Haddad. 2003. Predicting which species will benefit from corridors in fragmented landscapes from population growth models. American Naturalist 161:808-820.

Hurvich, C. M., and C. L. Tsai. 1989. Regression and timeseries model selection in small samples. Biometrika 76:297307.

Kolozsvary, M. B., and R. K. Swihart. 1999. Habitat fragmentation and the distribution of amphibians: patch and landscape correlates in farmland. Canadian Journal of Zoology 77:1288-1299.

Kuefler, D., N. M. Haddad, S. Hall, B. Hudgens, B. Bartel, and E. Hoffman. 2008. Distribution, population structure, and habitat use of the endangered St. Francis' satyr butterfly, Neonympha mitchellii francisci. American Midland Naturalist 159:298-320.
Kuefler, D., B. Hudgens, N. M. Haddad, W. F. Morris, and N. Thurgate. 2010. The conflicting role of matrix habitats as conduits and barriers for dispersal. Ecology 91:944-950.

Leidner, A. K., and N. M. Haddad. 2011. Combining measures of dispersal to identify conservation strategies in fragmented landscapes. Conservation Biology 25:1022-1031.

Levey, D. J., B. M. Bolker, J. J. Tewksbury, S. Sargent, and N. M. Haddad. 2005. Effects of landscape corridors on seed dispersal by birds. Science 309:146-148.

Lima, S. L., and P. A. Zollner. 1996. Towards a behavioral ecology of ecological landscapes. Trends in Ecology and Evolution 11:131-135.

Liu, C., P. M. Berry, T. P. Dawson, and R. G. Pearson. 2005. Selecting thresholds of occurrence in the prediction of species distributions. Ecography 28:385-393.

Martin, J., M. C. Runge, J. D. Nichols, B. C. Lubow, and W. L. Kendall. 2009. Structured decision making as a conceptual framework to identify thresholds for conservation and management. Ecological Applications 19:1079-1090.

McCarthy, M. A., and H. P. Possingham. 2007. Active adaptive management for conservation. Conservation Biology 21:956963.

McRae, B. H., and P. Beier. 2007. Circuit theory predicts gene flow in plant and animal populations. Proceedings of the National Academy of Sciences USA 104:19885-19890.

Phillips, S. J., R. P. Anderson, and R. E. Schapire. 2006. Maximum entropy modeling of species geographic ranges. Ecological Modelling 190:231-259.

R Development Core Team. 2005. R: a language and environment for statistical computing. R Foundation for Statistical Computing, Vienna, Austria.

Revilla, E., and T. Wiegand. 2008. Individual movement behavior, matrix heterogeneity, and the dynamics of spatially structured populations. Proceedings of the National Academy of Sciences USA 105:19120-19125.

Revilla, E., T. Wiegand, F. Palomares, P. Ferreras, and M. Delibes. 2004. Effects of matrix heterogeneity on animal dispersal: from individual behavior to metapopulation-level parameters. American Naturalist 164:E130-E153.

Ricketts, T. H. 2001. The matrix matters: effective isolation in fragmented landscapes. American Naturalist 158:87-99.

Ruckelshaus, M., C. Hartway, and P. Kareiva. 1997. Assessing the data requirements of spatially explicit dispersal models. Conservation Biology 11:1298-1306.

Schultz, C. B., and E. E. Crone. 2001. Edge-mediated dispersal behavior in a prairie butterfly. Ecology 82:1879-1892.

Wang, R., O. Ovaskainen, Y. Cao, H. Chen, Y. Zho, C. Xu, and I. Hanski. 2011. Dispersal in the Glanville fritillary butterfly in fragmented versus continuous landscapes: comparison between three methods. Ecological Entomology $36: 251-260$.

\title{
Supplemental Material
}

\author{
Appendix A \\ Comparison of movement behaviors among butterfly species (Ecological Archives A022-090-A1).
}

\section{Appendix B}

Calculating likelihoods, number of parameters, and marginal costs for butterfly dispersal models (Ecological Archives A022-090A2). 